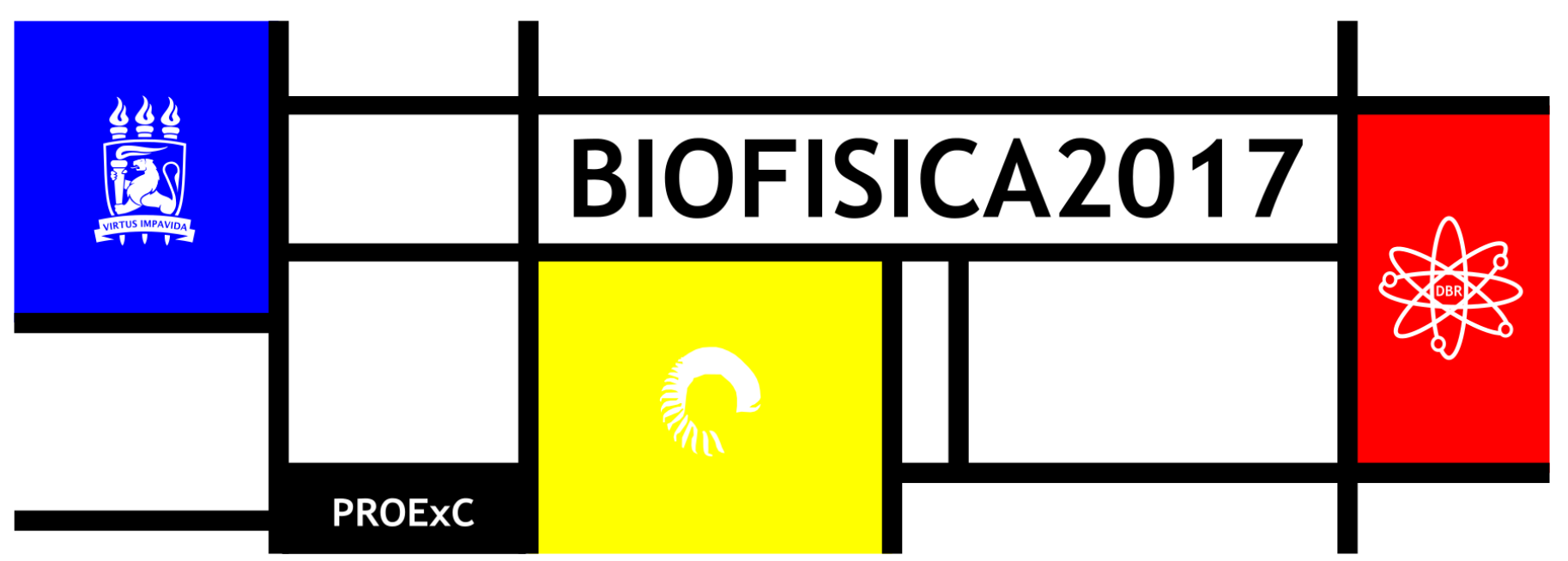

\title{
DISTRIBUIÇÃO DE MOLUSCOS TERRESTRES EM MANGUEZAIS DO NORDESTE BRASILEIRO
}

\author{
Julyanne Mélo ${ }^{1 *}$, Katarine Santos ${ }^{1}$, Kaio Freitas ${ }^{1}$, Ana Melo ${ }^{2}$, Elvis França ${ }^{1}$ \\ ${ }^{1} \mathrm{CRCN} / \mathrm{NE} ;{ }^{2}$ Laboratório de Biofísica e Radiobiologia, UFPE. \\ *julyanne.melo@ufpe.br
}

\section{INTRODUÇÃO}

Os biomonitores são organismos ou comunidades que respondem às alterações do ambiente, modificando suas funções vitais, ou sua composição química, permitindo verificar e avaliar a qualidade ambiental ou os efeitos da poluição ambiental. A escolha de um bom biomonitor de qualidade ambiental permite compreender facilmente a magnitude do estresse, características do habitat, ou ainda, o grau de exposição ao agente estressor e da resposta biológica à exposição. Embora o número de indicadores potenciais seja infinito, a seleção de alguns bons monitores não é simples (KLUMPP, 2001; OEHLMANN; SCHULTE-OEHLMANN, 2003; MAGALHÃES JÚNIOR, 2007; KAPUSTA, 2008).

Classificados como ecossistemas costeiros, os manguezais são ecossistemas de elevada importância ecológica e estão presentes em regiões tropicais e subtropicais, atuando como exportadores de matéria orgânica para os estuários, além de proteger as espécies ativas e favorecer a ciclagem de nutrientes e atuarem como absorvedores de poluentes. Dentre os ecossistemas brasileiros, é tido como um dos indicadores ambientais mais representativos de integridade da Zona Costeira, devido à vegetação característica e à variedade de nichos ecológicos, proporcionando um berçário de muitas espécies marinhas e o habitat de tantas outras, inclusive moluscos (SCHAEFFER-NOVELLI, 1995; ALVES, 2001; JESUS et al., 2004; ONOFRE et al., 2007; PRATES et al., 2010).

Espécies de moluscos terrestres como Littoraria angulifera (Lamarck, 1822) (Gastropoda: Littorinidae), Melampus coffea (Linnaeus, 1758) (Gastropoda, Ellobiidae) e Neritina virginea (Linnaeus, 1758) (Gastropoda: Neritidae) são encontrados nos manguezais, sendo principalmente encontrados na costa nordestina (MATTHEWS, 1970; MATTHEWS-CASCON; LOTUFO, 2006). Por sua expressiva população, essas espécies têm potencial para a aplicação como biomonitores. Com base nesta possibilidade, o objetivo deste trabalho foi avaliar a distribuição de moluscos terrestres das espécies Littoraria angulifera, Melampus coffea e Neritina virginea em manguezais das costas paraibana e potiguar.

\section{MATERIAIS E MÉTODOS}

Foram estudados 14 manguezais ao longo da costa dos Estados da Paraíba e Rio Grande do Norte conforme representa a Tabela 1. Em cada local, observaram-se suas características específicas onde foram coletados moluscos terrestres disponíveis na região.

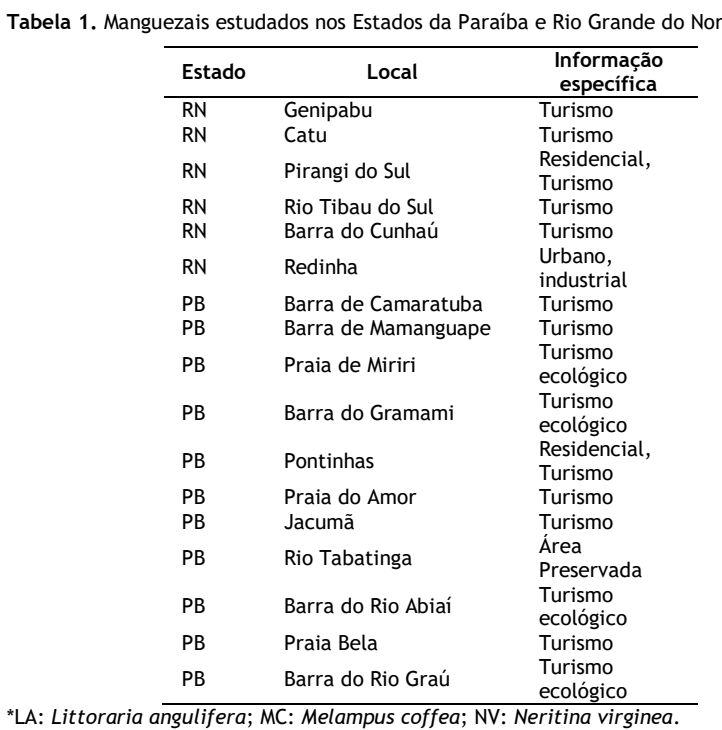

\section{RESULTADOS E DISCUSSÃO}

Embora não se tenha observado nenhuma relação significativa com relação à espécie Littoraria angulifera, houve uma tendência à

๑ 2017 CC BY-NC-ND LICENSE. ANAIS: ENCONTRO ANUAL DA BIOFÍSICA (2017): 85-87. BIOFÍSICA E RADIOBIOLOGIA, BIOCIÊNCIAS, UFPE $9 \mathrm{E} 10$ DE MARÇO, RECIFE, PERNAMBUCO, BRASIL DOI: 10.5151/biofisica2017-032 
predominância de indivíduos da espécie Melampus coffea em manguezais mais preservados e predominância populacional de Neritina virginea em manguezais urbanos do Rio Grande do Norte, conforme está apresentado na Tabela 2.

Tabela 2. Distribuição das espécies de moluscos terrestres avaliadas de acordo com a característica do manguezal estudado.

\begin{tabular}{|c|c|c|c|c|c|}
\hline Local & $\begin{array}{c}\text { Espécies } \\
\text { encontradas }\end{array}$ & Total & $\begin{array}{l}\text { L. } \\
\text { angulifera }\end{array}$ & $\begin{array}{l}\text { M. } \\
\text { coffea }\end{array}$ & $\begin{array}{l}N . \\
\text { virginea }\end{array}$ \\
\hline \multicolumn{6}{|l|}{$\mathrm{RN}$} \\
\hline Genipabu & LA, MC, NV & 135 & 66 & 34 & 35 \\
\hline Catu & $\mathrm{LA}, \mathrm{MC}$ & 93 & 77 & 16 & 0 \\
\hline Pirangi do Sul & $\mathrm{LA}, \mathrm{MC}$ & 85 & 65 & 20 & 0 \\
\hline Rio Tibau do Sul & LA & 79 & 79 & 0 & \\
\hline Barra do Cunhaú & LA, MC & 84 & 18 & 66 & 0 \\
\hline Redinha & $M C, N V$ & 108 & 0 & 27 & 81 \\
\hline Total (RN) & LA & 584 & 305 & 163 & 116 \\
\hline \multicolumn{6}{|l|}{ PB } \\
\hline $\begin{array}{c}\text { Barra de } \\
\text { Camaratuba }\end{array}$ & $\mathrm{LA}, \mathrm{MC}$ & 99 & 83 & 16 & 0 \\
\hline $\begin{array}{l}\text { Barra de } \\
\text { Mamanguape }\end{array}$ & LA & 111 & 111 & 0 & 0 \\
\hline Praia de Miriri & $\mathrm{LA}, \mathrm{MC}$ & 133 & 93 & 40 & 0 \\
\hline $\begin{array}{l}\text { Barra do } \\
\text { Gramami }\end{array}$ & $\mathrm{LA}, \mathrm{MC}$ & 173 & 84 & 89 & 0 \\
\hline $\begin{array}{l}\text { Gramami } \\
\text { Pontinhas }\end{array}$ & LA, MC & 81 & 67 & 14 & 0 \\
\hline Praia do Amor & $\mathrm{LA}, \mathrm{MC}$ & 147 & 100 & 47 & \\
\hline Jacumã & - & 0 & 0 & 0 & 0 \\
\hline Rio Tabatinga & - & 0 & 0 & 0 & 0 \\
\hline Barra do Rio & $\mathrm{LA}, \mathrm{MC}$ & 99 & 22 & 77 & 0 \\
\hline $\begin{array}{l}\text { Abiaí } \\
\text { Praia Bela }\end{array}$ & LA, MC & 0 & 0 & 0 & 0 \\
\hline $\begin{array}{l}\text { Barra do Rio } \\
\text { Graú }\end{array}$ & - & 120 & 86 & 34 & 0 \\
\hline Total (PB) & & 963 & 646 & 317 & 0 \\
\hline Total & & 1547 & 951 & 480 & 116 \\
\hline
\end{tabular}

A distribuição dos animais nos locais estudados estão representados na Figura 1 para o Estado do Rio Grande do Norte e Figura 2 para o Estado da Paraíba.

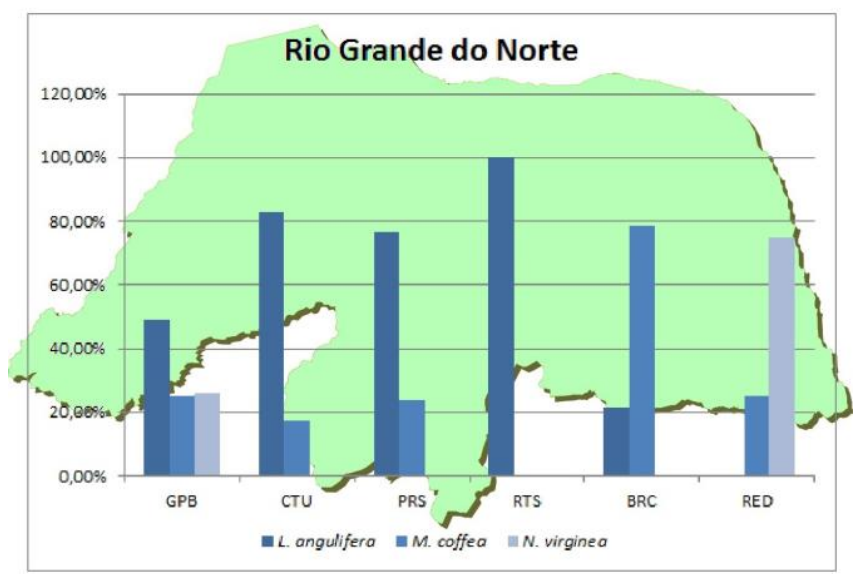

Figura 1. Distribuição das espécies no Rio Grande do Norte. GPB: Genipabu; CTU: Catu; PRS: Pirangi do Sul; RTS: Rio Tibau do Sul; BRC: Barra do Cunhaú; RED: Redinha.

A espécie Neritina virginea foi observada apenas nos manguezais do Rio Grande do Norte. Exemplares desta espécie são encontrados no Ceará, Rio Grande do Norte, São Paulo e manguezais da Região Sul, além de Belize, Mar do Caribe, Colômbia, Costa Rica, Cuba, Golfo do México, Jamaica, México, Panamá e Venezuela (MATTHEWS-CASCON; LOTUFO, 2006; WoRMS, 2017).

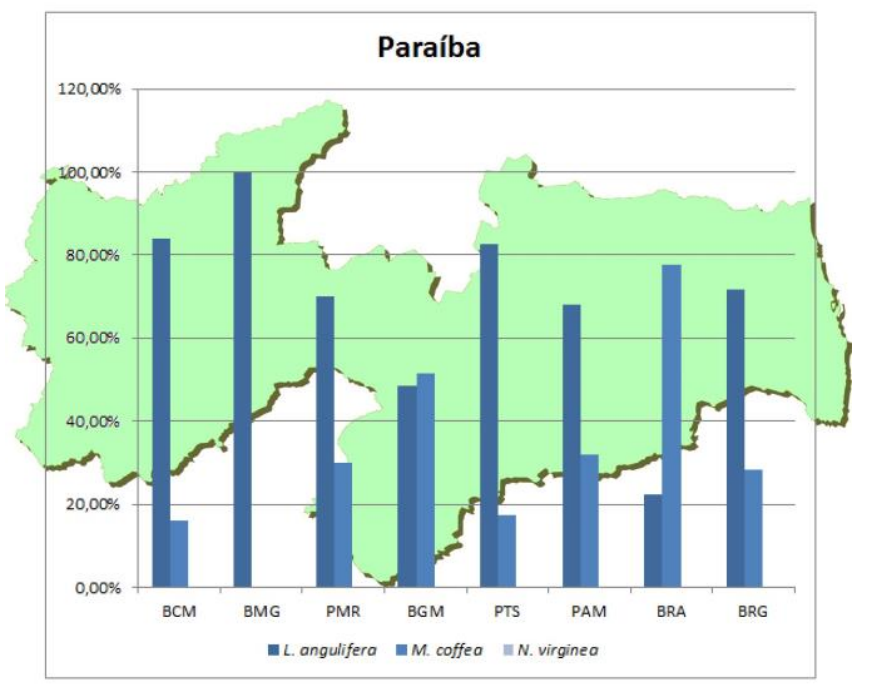

Figura 2. Distribuição das espécies na Paraíba. BCM: Barra de Camaratuba; BMG: Barra de Mamanguape; PMR: Praia de Miriri; BGM: Barra do Gramami; PTS: Pontinhas; PAM: Praia do Amor; BRA: Barra do Rio Abiaí; BRG: Barra do Rio Graú.

\section{CONCLUSÕES}

Considerando-se o mesmo tempo de coleta para a obtenção das amostras e que o número de animais amostrados é proporcional ao número total de animais de cada espécie, pode-se concluir que:

A espécie Littoraria angulifera é a espécie predominante na maioria dos manguezais observados, entretanto, não foram observadas correlações significantes entre distribuição e características locais.

A espécie Neritina virginea apresentou distribuição interessante em manguezal urbano do Rio Grande do Norte.

Moluscos terrestres da espécie Melampus coffea possibilitaram algumas associações por estarem presentes nos manguezais dos dois Estados.

Estudos complementares são necessários para melhor avaliação dos parâmetros específicos e análise mais detalhada de informações específicas para cada local estudado.

\section{REFERÊNCIAS}

ALVES, J. R. P. Manguezais: educar para proteger. Rio de Janeiro: FEMAR: SEMADS, 2001, 96 p.

JESUS, H. C.; COSTA, E. A.; MENDONÇA, A. S. F.; ZANDONADE, E. Distribuição de metais pesados em sedimentos do sistema estuarino da ilha de Vitória-ES. Química Nova, v. 27, p. 378-386, 2004.

KAPUSTA, S. C. Bioindicação ambiental - Curso Técnico em Meio Ambiente. Porto Alegre: Escola Técnica da Universidade Federal do Rio Grande do Sul, 2008.

KLUMPP, A. Utilização de bioindicadores de poluição em condições temperadas e tropicais. In: MAIA, N.B.; MARTOS, H. L.; BARRELLA, W. (Org.). Indicadores ambientais: conceitos e aplicações. São Paulo: EDUC - Editora da PUC. p. 77-94, 2001.

MAGALHÃES JÚNIOR, A. P. Indicadores ambientais e recursos hídricos: realidade e perspectivas para o Brasil a partir da experiência francesa. Rio de Janeiro: Bertrand Brasil, 688 p., 2007. 
MATTHEWS-CASCON, H.; LOTUFO, T. M. C. Biota marinha da costa oeste do Ceará. Série Biodiversidade, v. 24, Brasília: MMA, 2006, 248p.

MATTHEWS, H. R. Moluscos marinhos do norte e nordeste do Brasil II - Moluscos do Arquipélago de Fernando de Noronha (com algumas referências ao Atol das Rocas). Arquivos de Ciências do Mar, v. 10, p. 1-53, 1970.

MURAYAMA, R. K. Ecologia populacional do gastropoda Neritina virginea (Linnaeus, 1758) na zona entremarés da Baía do Araçá, litoral norte de São Paulo. Dissertação (mestrado). Universidade Estadual de Campinas - UNICAMP, 2016.

OEHLMANN, J.; SCHULTE-OEHLMANN, U. Mollusks as bioindicators. In: MARKET, B. A.; BREURE, A. M.; ZECHMEISTER, H. G. Bioindicators and biomonitors: principles, concepts and applications, London: Elsevier, 2003, p. 577-635.

ONOFRE, C. R. E.; CELINO, J. J.; NANO, R. M. W.; QUEIROZ, A. F. S. Biodisponibilidade de metais traços nos sedimentos de manguezais da porção norte da Baía de Todos os Santos, Bahia, Brasil. Revista de Biologia e Ciências da Terra, v. 2, p. 65-82, 2007.

PRATES, A. P.; GONÇALVES, M. A; ROSA, M. Panorama da Conservação dos ecossistemas Costeiros e Marinhos no Brasil. 2 ed. rev. ampliada - Ministério do Meio Ambiente. Brasília, MMA, 2010 .

SCHAEFFER-NOVELLI, Y. Manguezal: Ecossistema entre a terra e o mar. $1^{\circ}$ ed. São Paulo: Caribbean Ecological Research, 64p. 1995. WoRMS. Neritina virginea. World Register of Marine Species, 2017. Disponivel em: <http://www.marinespecies.org/ aphia.php?p=taxdetails\&id=419512> Acesso em: 04 de fevereiro de 2017. 